\title{
Synchronization by Food Access Modifies the Daily Variations in Expression and Activity of Liver GABA Transaminase
}

\author{
Dalia De Ita-Pérez, Isabel Méndez, Olivia Vázquez-Martínez, Mónica Villalobos-Leal, and \\ Mauricio Díaz-Muñoz
}

Instituto de Neurobiología, Campus UNAM-Juriquilla, 76230 Querétaro, QRO, Mexico

Correspondence should be addressed to Mauricio Díaz-Muñoz; mdiaz@comunidad.unam.mx

Received 13 December 2013; Revised 3 March 2014; Accepted 11 March 2014; Published 7 April 2014

Academic Editor: Mario Guido

Copyright (C) 2014 Dalia De Ita-Pérez et al. This is an open access article distributed under the Creative Commons Attribution License, which permits unrestricted use, distribution, and reproduction in any medium, provided the original work is properly cited.

\begin{abstract}
Daytime restricted feeding (DRF) is an experimental protocol that influences the circadian timing system and underlies the expression of a biological clock known as the food entrained oscillator (FEO). Liver is the organ that reacts most rapidly to food restriction by adjusting the functional relationship between the molecular circadian clock and the metabolic networks. $\gamma$ Aminobutyric acid (GABA) is a signaling molecule in the liver, and able to modulate the cell cycle and apoptosis. This study was aimed at characterizing the expression and activity of the mostly mitochondrial enzyme GABA transaminase (GABA-T) during DRF/FEO expression. We found that DRF promotes a sustained increase of GABA-T in the liver homogenate and mitochondrial fraction throughout the entire day-night cycle. The higher amount of GABA-T promoted by DRF was not associated to changes in GABA-T mRNA or GABA-T activity. The GABA-T activity in the mitochondrial fraction even tended to decrease during the light period. We concluded that DRF influences the daily variations of GABA-T mRNA levels, stability, and catalytic activity of GABA-T. These data suggest that the liver GABAergic system responds to a metabolic challenge such as DRF and the concomitant appearance of the FEO.
\end{abstract}

\section{Introduction}

GABA transaminase (GABA-T; 4-aminobutanoate:2-oxoglutarate aminotransferase, EC 2.6.1.19) is a catabolic enzyme that converts GABA and $\alpha$-ketoglutarate into succinate semialdehyde and glutamate. Its specificity is not strict since it can also recognize alanine, $\beta$-alanine, aspartate, and propanoate as substrates. GABA-T is dependent on pyridoxal-phosphate (PLP) as cofactor, and its catalytic mechanism consists of two coupled half reactions in which the PLP cofactor oscillates between the pyridoxal and the pyridoxamine forms [1]. GABA-T has been found in a large variety of species ranging from microorganisms, plants, invertebrates, and vertebrates. In eukaryotic cells, GABA-T is present, but not exclusively, within the mitochondria [2, 3]. This enzyme has been crystallized from several sources. The structure of GABA-T from brain pig indicates a $\alpha 2$ dimer with 472 residues per subunit; the two monomers are tightly intertwined, and the two PLP cofactors are located close to the subunit interface [4]. The structure of the brain GABA$\mathrm{T}$ also contains a $[2 \mathrm{Fe}-2 \mathrm{~S}]$ cluster in the vicinity of the PLP cofactors [5]. The maturation process in the synthesis of GABA-T involves a proteolytic cleavage that removes the $\mathrm{XRX}(*) \mathrm{XS}$ motif. However, in the liver, GABA-T is further cleaved to a smaller isoform by a second proteolytic step catalyzed by a mitochondrial processing peptidase [6].

GABA is a well-known inhibitory transmitter in the nervous system. However, components of the GABAergic system have also been characterized in a variety of endocrine tissues such as pancreas, testis, and liver [7-9]. Mammalian liver contains GABA at nM levels as well as its specific transporters (GAT2) and receptors (both GABAR-A and GABAR-B) [10]. Liver GABA has been proposed to function as a positive regulator of the cell cycle, with implications in some forms of hepatocellular carcinoma [11]. High GABA-T activity has been reported in hepatic tissue [12], the rationale being that liver GABA-T mainly functions to degrade 
the GABA produced by microorganisms in the gastrointestinal tract [13]. In addition, GABA can also be present in the food ingested at mealtime.

Daytime restricted feeding (DRF) is an experimental protocol that influences the circadian timing system by promoting the emergence of an alternate master circadian oscillator that is independent of the suprachiasmatic nucleus (SCN) [14]. The basis for this new form of measuring the "physiological timing" is a circadian entity known as the food entrainable oscillator (FEO), but its anatomical location is still unidentified [15]. The DRF protocol involves the establishment of a catabolic response promoted by limiting the food intake to a few hours $(2-4 \mathrm{~h})$ during the light phase of the day-night cycle. The display of an anticipatory behavior is evident before mealtime (food anticipatory activity, FAA) as is a diversity of physiological and metabolic adaptations associated with DRF/FEO expression [16]. During the DRF protocol, some biochemical and structural changes in the liver have been associated with improved mitochondrial function as well as increased apoptotic activity and cell division, resulting in enhanced cellular exchange $[17,18]$.

To obtain further insights into the modulation of the mitochondrial adaptations of the liver GABA-handling system during the DRF protocol/FEO expression, we analyzed the daily variations of GABA-T mRNA and protein expression and enzymatic activity. The results evidenced under the DRF protocol are as follows: (1) an increased presence of GABA-T in homogenate and mitochondrial fraction, (2) changes in the rhythmic profile of GABA-T activity, and (3) disappearance of an ultradian rhythm of GABA-T mRNA.

\section{Materials and Methods}

2.1. Animals and Housing. Experiments were carried out with male Wistar rats weighing from 200 to $250 \mathrm{~g}$ and maintained under a 12:12 h LD cycle (lights on 08:00 h) at constant temperature $\left(22 \pm 1^{\circ} \mathrm{C}\right)$. Rats were kept in groups of 4 in transparent acrylic cages $(40 \times 50 \times 20 \mathrm{~cm})$ with free access to Purina Chow and water except during food restriction or fasting conditions. Experimental procedures were conducted in accordance with our Institutional Guide for Care and Use of Experimental Animals (Universidad Nacional Autónoma de México) and with international ethical standards [19].

2.2. Experimental Design. The experimental protocol has been published previously [20]. Briefly, rats were randomly assigned to one of the following feeding conditions for 3 weeks:

(1) control animals fed with ad libitum with free access to food and water throughout the $24 \mathrm{~h}$ period, AL group;

(2) experimental group with restricted food access (exclusively from 12:00 to 14:00 h), DRF group.

At the end of the 3 weeks, subgroups of animals were sacrificed at $3 \mathrm{~h}$ intervals over a complete $24 \mathrm{~h}$ cycle, starting at 08:00 h. The protocol was followed until the last day.
In addition, 2 more groups were included to compare the acute fasting and subsequent refeeding response in the DRF group.

(1) Animals fed ad libitum were maintained with free food access for 3 weeks; on the last day, food was removed at 14:00 h, and, after $21 \mathrm{~h}(\sim 1$ day) of food deprivation, they were sacrificed (at 11:00 h), F group.

(2) A second group of rats was similarly deprived of food for $21 \mathrm{~h}$, then refed for $2 \mathrm{~h}$ (from 12:00 to 14:00), and sacrificed at 14:00 h, F + R group.

2.3. Liver Sampling and Subcellular Fractionation. Rats were beheaded with a guillotine-like instrument. The liver was removed $(\approx 5 \mathrm{~g})$ and immediately placed in an ice-cold isolation medium ( $1: 10 \mathrm{wt} / \mathrm{vol})$ containing $250 \mathrm{mM}$ sucrose, $0.1 \%$ BSA (fatty acid free), and $0.5 \mathrm{mM}$ EGTA (pH 7.4). The tissue was homogenized in 10 volumes of $10 \mathrm{mM}$ Tris- $\mathrm{HCl}, \mathrm{pH}$ 7.4, with a Teflon homogenizer ( $40 \mathrm{rpm}$ for $10 \mathrm{~s}$ ). Subcellular fractionation was done according to Aguilar-Delfín et al. [21]. Briefly, the homogenate was centrifuged at 1,500 g for $15 \mathrm{~min}$, and the supernatant was centrifuged at $10,000 \mathrm{~g}$ for $20 \mathrm{~min}$ to sediment the mitochondrial fraction. Protein was measured by the method of Bradford [22].

2.4. GABA-T Activity. GABA-T activity was measured by a coupled enzymatic assay according to the method reported by Jung et al., 1977 [23]. The method involves the conversion of GABA to succinic acid by the consecutive reactions of GABA$\mathrm{T}$ (in the sample) and semialdehyde dehydrogenase (added in the assay). As part of the reactions, $\mathrm{NAD}^{+}$is reduced to $\mathrm{NADH}$, allowing the quantification of GABA transamination by spectrophotometric recording at $340 \mathrm{~nm}$. The increasing in optical density was recorded during the first 2 min of the assay.

2.5. Western Blot Analysis. Liver homogenates and mitochondrial fractions were obtained in RIPA buffer (SigmaAldrich, SLM, USA) and subjected to denaturing SDS-PAGE under reducing conditions. Total protein concentrations were determined by the Bradford method and equal amounts $(30 \mu \mathrm{g})$ were separated on $10 \%$ SDS-PAGE, transferred to nitrocellulose membranes, and blocked for $1 \mathrm{~h}$ in TBST buffer (20 mM TRIS, pH 7.5; 500 mM NaCl; 0.5\% Tween 20) containing 5\% nonfat milk. Membranes were then washed and incubated in the presence of mouse anti-GABA-T antibody ( $\mathrm{Rb} \mathrm{mAb}$ to ABAT, ab108249, Abcam, Cambridge, UK) diluted $1 / 10,000$ in TBST overnight at $4^{\circ} \mathrm{C}$. As controls, in the case of homogenates, membranes were incubated in the presence of mouse anti-tubulin antibody (ab56676, Abcam, Cambridge, UK) diluted $1 / 1,000$ or in the case of mitochondrial fractions in the presence of rabbit anti-VDAC1/Porin antibody (ab15895, Abcam, Cambridge, UK) diluted 1/1,000. After washing, membranes were incubated with secondary antibodies conjugated to alkaline phosphatase $(1 / 5,000)$. Bands were revealed using AP conjugate substrate kit (BioRad, CA, USA). Densitometric analysis was performed using the Image Lab Software (v 3.0, Bio-Rad, CA, USA). 
AL $\quad \mathrm{Hg} \quad \mathrm{DRF}$
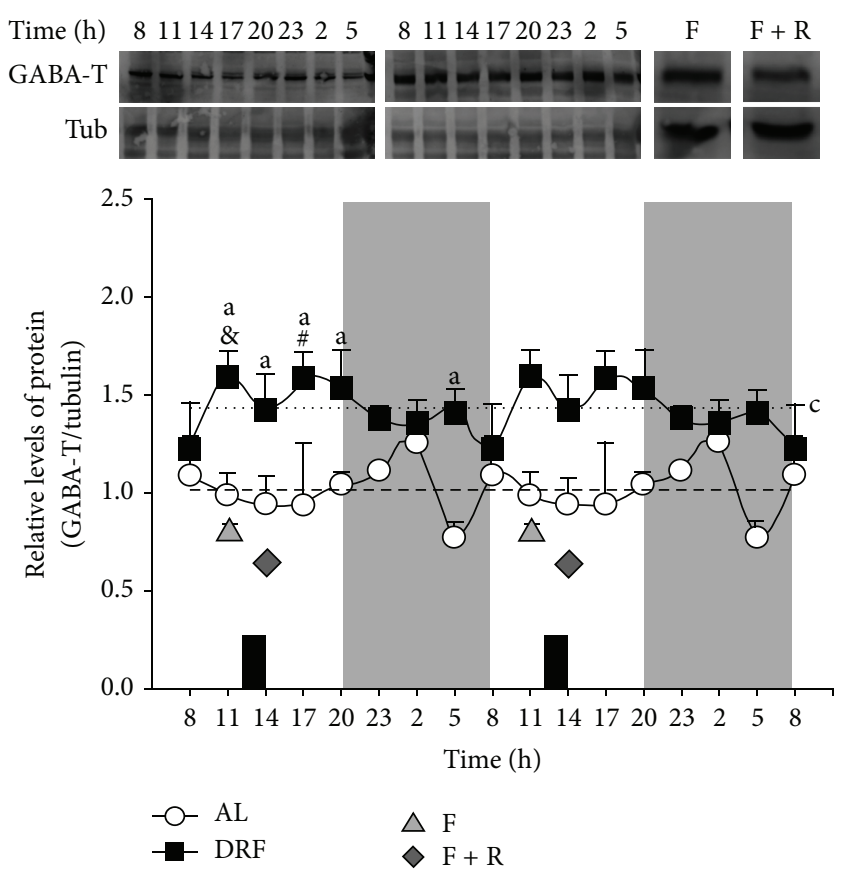

(a)
Mt

$\mathrm{AL}$

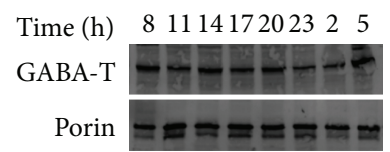

DRF
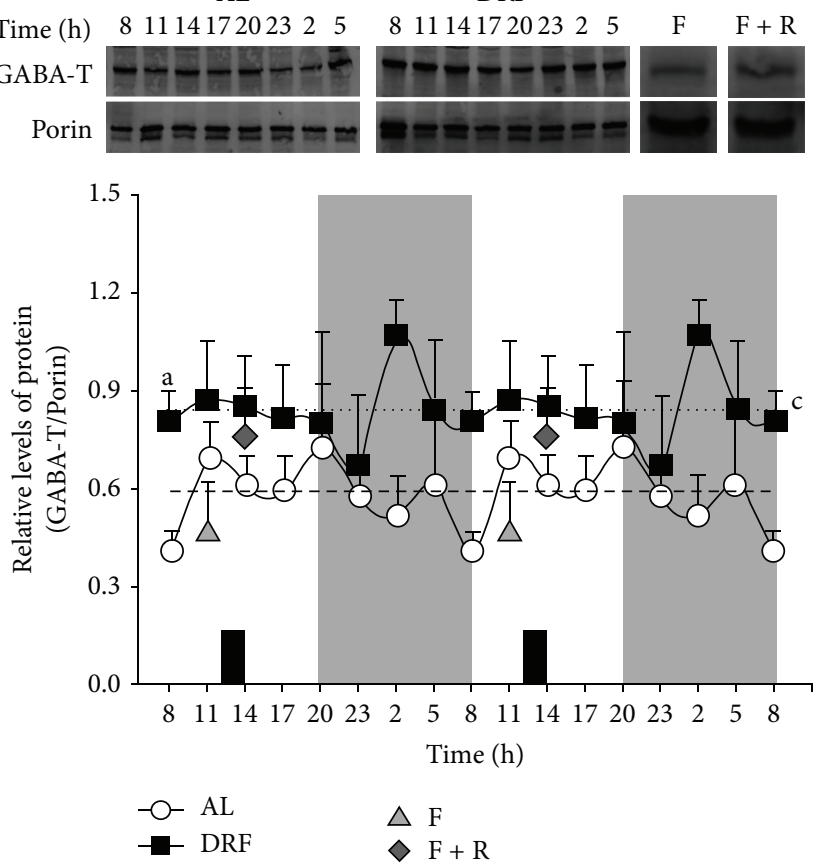

(b)

FIGURE 1: Western blot analysis of $24 \mathrm{~h}$ profile of GABA-T protein in liver of AL and DRF rats. Liver homogenates (Hg) (a) and mitochondrial fractions (Mt) (b) were subjected to electrophoresis on 10\% SDS-polyacrylamide gels. Data were quantified by densitometry of the bands obtained from homogenates (a) and mitochondrial fractions (b) collected over a $24 \mathrm{~h}$ period from the AL and DRF groups and food condition controls, F and F + R. Food availability for the DRF group is indicated by dark boxes (from 12:00 to 14:00 h). Average values are represented as a dashed line for the AL group and as a dotted line for the DRF group. Graphs represent the mean \pm SEM of 4 rats per time point. Significant differences $(P<0.05)$ are indicated as follows: a: DRF versus AL by two-way ANOVA and post hoc Bonferroni test; c: mean of DRF versus mean of AL by Student's $t$-test for both homogenate and mitochondrial fraction; for homogenates only: \&, DRF versus F at 11:00; \#, DRF versus $\mathrm{F}+\mathrm{R}$ at 14:00, both by the Student's $t$-test.

TABLE 1: Chronobiological analysis of liver GABA-T parameters: mRNA, protein amount, and activity.

\begin{tabular}{|c|c|c|c|c|c|c|c|c|c|c|}
\hline & \multirow{2}{*}{\multicolumn{2}{|c|}{ GABA-T mRNA }} & \multicolumn{4}{|c|}{ GABA-T protein } & \multicolumn{4}{|c|}{ GABA-T activity } \\
\hline & & & \multicolumn{2}{|c|}{$\mathrm{Hg}$} & \multicolumn{2}{|c|}{$\mathrm{Mt}$} & \multicolumn{2}{|c|}{$\mathrm{Hg}$} & \multicolumn{2}{|c|}{$\mathrm{Mt}$} \\
\hline & $\mathrm{AL}$ & DRF & $\mathrm{AL}$ & DRF & $\mathrm{AL}$ & DRF & $\mathrm{AL}$ & DRF & $\mathrm{AL}$ & DRF \\
\hline Rhythm (\%) & - & - & - & - & - & - & - & 54.8 & 40.3 & 38.4 \\
\hline Mesor & 0.9 & 0.8 & 1.1 & 1.5 & 0.7 & 0.9 & 5.2 & 5.4 & 2.4 & 2.0 \\
\hline Amplitude & 0.3 & - & - & - & - & - & - & 1.55 & 0.48 & 0.50 \\
\hline Acrophase (h:min) & $7: 40 / 15: 15 / 22: 50$ & - & - & - & - & - & - & $9: 56$ & $5: 56$ & 4:14 \\
\hline Period & $8 \mathrm{~h}$ & - & - & - & - & - & - & $24 \mathrm{~h}$ & $24 \mathrm{~h}$ & $24 \mathrm{~h}$ \\
\hline
\end{tabular}

Chronos-Fit analysis was performed to evaluate rhythmicity for GABA-T parameters in liver. Acrophases in GABA-T mRNA from AL group were repeated each $7 \mathrm{~h}$ with $40 \mathrm{~min}$. Hg: liver homogenate, Mt: mitochondrial fraction, AL: ad libitum, and DRF: daytime restricted feeding. (-) means that no rhythmic pattern was detected.

2.6. RT-qPCR Amplifications. GABA-T gene expression was evaluated by isolating total RNA from liver tissues (20-30 mg) using the SV Total RNA Isolation System (Promega, WI, USA). The amount and quality of RNA were estimated spectrophotometrically at 260 and $280 \mathrm{~nm}$, and a constant amount of RNA $(2 \mu \mathrm{g})$ was reverse transcribed using SuperScript III Reverse Transcriptase, Oligo (dT) 12-18 Primer, RNaseOUT Recombinant Ribonuclease Inhibitor, and dNTP Set PCR Grade (Invitrogen, CA, USA). Amplification was performed in triplicate in the CFX96TM
Real-Time PCR Detection System (Bio-Rad, CA, USA). Primers used for qPCR amplifications were synthesized by Sigma-Aldrich Co. (MO, USA), and the corresponding sequences were, for GABA-T (GenBank BC081787.1), forward $5^{\prime}$-TTCCGGAAGCTGAGAGACAT- $3^{\prime}$ and reverse $5^{\prime}$-AGTCTGAACCTCGTCCACCA-3' and, for ribosomal protein S18 (Rps18) (GenBank BC126072.1) used as housekeeping gene, forward 5'-TTCAGCACATCCTGCGAGTA- $3^{\prime}$ and reverse $5^{\prime}$-TTGGTGAGGTCAATGTCTGC$3^{\prime}$. Amplifications were carried out with Maxima SYBR 
Green qPCR Master Mix (Thermo Fisher Scientific, MA USA) in a $10 \mu \mathrm{L}$ final reaction volume containing cDNA (1/100) and $0.5 \mu \mathrm{M}$ of each of the primer pairs in SYBR Green Master Mix, according to the following protocol: activation of Taq DNA polymerase and DNA denaturation at $95^{\circ} \mathrm{C}$ for $10 \mathrm{~min}$, followed by 40 amplification cycles consisting of $10 \mathrm{~s}$ at $95^{\circ} \mathrm{C}, 30 \mathrm{~s}$ at $62^{\circ} \mathrm{C}$, and $30 \mathrm{~s}$ at $72^{\circ} \mathrm{C}$. The PCR data were analyzed by the $2^{-\Delta \Delta \mathrm{CT}}$ method and cycle thresholds (CT) normalized to the housekeeping gene Rps18 were used to calculate the mRNA levels of GABA-T.

2.7. Data Analysis. Data were classified by group and time and are displayed as mean \pm standard error of the mean (SEM). Data were compared using a two-way ANOVA using Fisher's least significant difference (LSD) test for multiple and independent measures, with a factor for group (2 levels) and a factor for time (8 levels). In order to determine significant time effects for each daily sampling profile, a one-way ANOVA was performed for individual groups. The one-way ANOVA was followed by Tukey's post hoc test whereas the two-way ANOVA was followed by a Bonferroni post hoc test with the significance threshold set at $P<0.05$ for both. The Student's $t$-test was applied to detect significant differences between DRF rats and the controls of feeding conditions (acute fasting and refeeding) both before and after food access (11:00 $\mathrm{h}$ and 14:00 h, resp.). The Student's $t$-test was also used to identify significant differences $(P<0.05)$ between the $24 \mathrm{~h}$ average values of the AL and DRF groups. Rhythmic analysis was performed by Chronos-Fit (v 1.06 developed by P. Zuther, S. Gorbey, and B. Lemmer, 2009) based on a partial Fourier analysis of the data [24]. All statistical analysis was performed with the program STATISTICA, version 4.5 (StatSoft Inc.).

\section{Results}

All results are double plotted displayed to have a better notion of the rhythmic profiles.

3.1. Liver GABA-T Is Increased by DRF. The amount of GABA-T protein detected by Western blot analysis in the liver homogenate and mitochondrial fraction is shown in Figure 1. GABA-T did not show any rhythmicity in liver homogenate from AL rats (a). DRF rats showed a significant increase in GABA-T protein throughout the $24 \mathrm{~h}$ cycle. The average value was $\sim 38 \%$ higher in the DRF group than in the AL group (Table 1 and Figure 1(a)). In addition, significant differences (higher levels in DRF than AL rats) were detected at 05:00 h, 11:00 h, 14:00 h, 17:00 h, and 20:00 h. Similar to the AL rats, no rhythmicity was detected in the Western blot signal in the homogenate of the DRF group. The level of GABA-T was responsive to acute $24 \mathrm{~h}$ fasting (F group), showing a significant reduction in comparison to the DRF group at 11:00 h. Two hours of feeding after fasting $(F+R$ group) were not enough to restore the low levels of GABA-T associated with the acute fasting condition (a).

Since GABA-T is in an important proportion a mitochondrial enzyme, the amount of this protein was also evaluated by Western blot analysis in the liver mitochondrial fraction in the AL and DRF groups (b). As observed in liver homogenate, GABA-T was significantly increased in the DRF group. However, comparing the calculated average values, the elevation was more moderate ( $26 \%$, Table 1$)$. Again, no rhythmicity was detected in either group. A significant difference between both groups was detected at 02:00 h. In contrast to the effect observed in the liver homogenate, neither acute fasting (group F) nor acute fasting followed by $2 \mathrm{~h}$ of refeeding ( $\mathrm{F}+\mathrm{R}$ group) modified the amount of GABA$\mathrm{T}$ protein in the mitochondrial fraction. However, as to the controls of acute feeding conditions, there was a significant increase $(\sim 85 \%)$ in the $\mathrm{F}+\mathrm{R}$ rats in comparison to the $\mathrm{F}$ rats.

\subsection{The Activity of Liver GABA-T in the Mitochondrial} Fraction Was Modified by DRF. The activity of GABA-T in AL and DRF groups is shown in Figure 2 for the liver homogenate (a) and for the mitochondrial fraction (b). GABA-T activity in the homogenate fluctuated in both groups, with peaks at 23:00 h and 08:00 h in AL and DRF rats, respectively (Table 1). At these times, significant differences were detected between both groups. In contrast, GABA-T activity in the mitochondrial fraction tended to be reduced in the DRF group, and both groups showed rhythmicity. Peak value for $\mathrm{AL}$ rats was at $08: 00 \mathrm{~h}$ and for DRF rats was at 05:00 h (Table 1). A clear sinusoidal pattern was observed in the AL group with the lowest value in the middle of the light phase and the peak at the transition from the dark and to the light period. The DRF group also exhibited a sinusoidal pattern with the lowest value near the end of the light period and the peak $12 \mathrm{~h}$ later. However, this pattern was consistent with the lower values of mitochondrial GABA-T activity, especially during the light period.

In both liver homogenate and the mitochondrial fraction, the food condition controls ( $\mathrm{F}$ and $\mathrm{F}+\mathrm{R}$ groups) did not show any changes in comparison to the AL and DRF rats at 11:00 h and 14:00 h, respectively.

3.3. mRNA of Liver GABA-T Was Not Rhythmic in the $D R F$ Group. The mRNA for GABA-T was quantified in liver by RT-qPCR in the AL and DRF groups, and the results are shown in Figure 3. AL rats showed rhythmicity in this parameter with 2 larger peaks, one at 17:00 h and the second at 23:00 h, and a minor peak at 08:00 h. There was also a pronounce valley at 20:00 h. Indeed, rhythmic analysis detected an ultradian rhythm with a period of $\sim 8 \mathrm{~h}$ (Table 1). DRF rats did not show any rhythmicity. Significant differences between AL and DRF groups were detected at 14:00 h, 17:00 h, and 23:00 h, being higher the values from the AL rats. No significant differences in the expression of GABA$\mathrm{T}$ mRNA were detected between the $\mathrm{F}$ and DRF group at 11:00 $\mathrm{h}$ as well as between the $\mathrm{F}+\mathrm{R}$ and DRF group at 14:00 $\mathrm{h}$.

\section{Discussion}

4.1. Liver GABAergic System. Our data show that under a protocol of daytime restricted feeding (DRF) with the subsequent appearance of the feeding entrained oscillator (FEO) the properties of liver GABA-T are affected and highlight the 


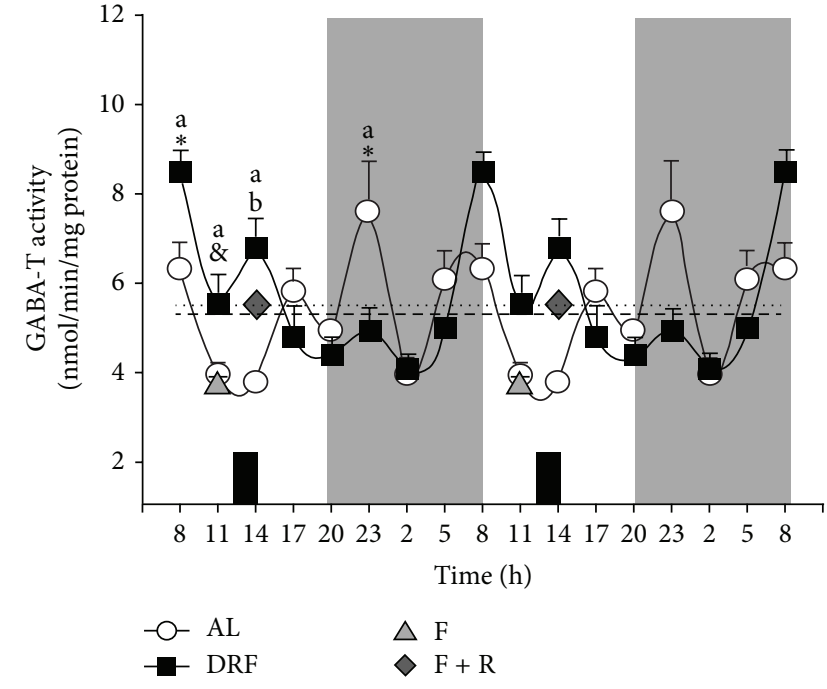

(a)

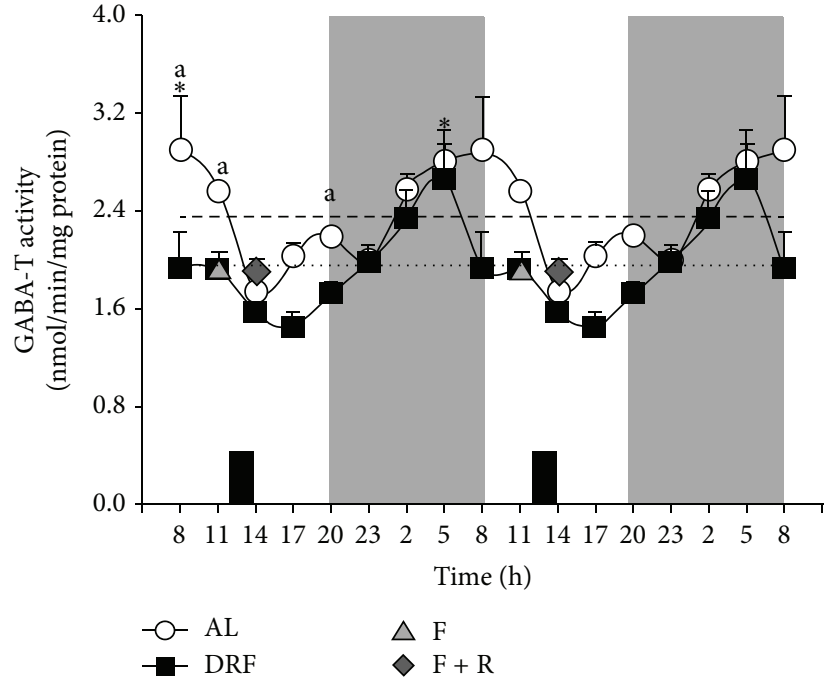

(b)

FIGURE 2: Analysis of $24 \mathrm{~h}$ profile of GABA-T activity in the liver of AL and DRF rats. GABA-T activity was measured in both the homogenate (a) and mitochondrial fraction (b) of the livers from AL and DRF rats by a spectrophotometric assay over a $24 \mathrm{~h}$ period. Rats were subjected to a $12 \mathrm{~h}: 12 \mathrm{~h}$ regime of light: dark. The shaded zone represents the dark phase. Food availability for RFS group is indicated by dark boxes (from 12:00 to 14:00 h). Controls of food condition are shown (F and F + R). Average values are represented as dashed line for AL group and a dotted line for RFS group. Graphs show the mean \pm SEM of 4 rats per time point. Significant differences $(P<0.05)$ are indicated as follows: *: DRF versus AL by one-way ANOVA and post hoc Bonferroni test, in both the homogenate and mitochondrial fraction; a: RFS versus AL by two-way ANOVA and post hoc Bonferroni test in the mitochondria fraction only; b: F versus F + R by Student's $t$-test.

plasticity of the hepatic GABAergic system under a metabolic challenge.

It is well documented that GABA and its metabolizing enzymes are found outside the nervous system and play a role in the cell signaling of several organs and tissues [25]. These tissues express the anabolic and catabolic enzymes for GABA, and measurable levels of this molecule can be detected $[7,12]$. It has also been reported that hepatic tissue expresses elements of the GABAergic system, such as specific receptors (GABAR-A and GABAR-B), transporters, and the catabolic enzyme GABA-T [10]. Hepatocytes and other liver cell types are clearly in contact with GABA, which may be produced by the liver itself or may come from the microbial production of the intestinal tract $[13,25]$. In this context, it has been suggested that GABA present in hepatic tissue could act as a regulator of the cell cycle and the migration of cancerous hepatocytes $[11,26]$. As a signaling molecule, liver GABA must be finely controlled by plasma membrane transporters but, mainly, by its catabolic transformation by GABA-T [27].

4.2. Liver GABA Transaminase. GABA-T is, but not exclusively, a mitochondrial enzyme that requires PLP for its activity. In the liver, it exists as a smaller variant because of proteolytic processing that removes 12-14 amino acids [6]. The pharmacological inhibition of GABA-T has been explored for several years, motivated by attempts to find an effective antiepileptic drug [28]; however, not much information has been reported regarding its transcriptional regulation or the modulation of its activity by covalent modifications.
Our results indicate that liver GABA-T activity, mainly in the mitochondrial fraction, and the level of GABA-T mRNA in AL groups show daily fluctuations. Previously, our group reported that the mitochondrial yield after cellular fractionation did not change by the DRF protocol [17]. The data also show that the DRF protocol affected the properties of hepatic GABA-T at different levels. (1) The elevated levels of this protein enzyme in the liver homogenate and mitochondrial fraction (Figure 1) did not correlate with the corresponding levels of mRNA (Figure 3). A possible explanation is that the restricted feeding schedule promoted an increased stability of GABA-T or an increment in its translational rate. (2) The higher amount in the presence of GABA-T did not correlate with the GABA-T activity measured in liver homogenate and the mitochondrial fraction (Figure 2). This lack of correspondence could be explained by the existence of a negative regulator of GABA-T activity, such as a covalent modification or some other type of posttranslational processing. We have not yet found any reports of phosphorylation, acetylation, methylation, or other modulatory modifications of GABA-T activity. However, these modifications in GABA-T properties promoted by the protocol of DRF/FEO expression can be considered as a metabolic adaptation based on the biochemical plasticity of this enzymatic system.

4.3. GABA-T under Food Synchronization/Food Entrainable Oscillator. The changes in the amount and activity of GABA$\mathrm{T}$ associated with the protocol of daytime restricted feeding are part of an extensive set of metabolic and physiological adaptations that occur in the liver and other organs when 


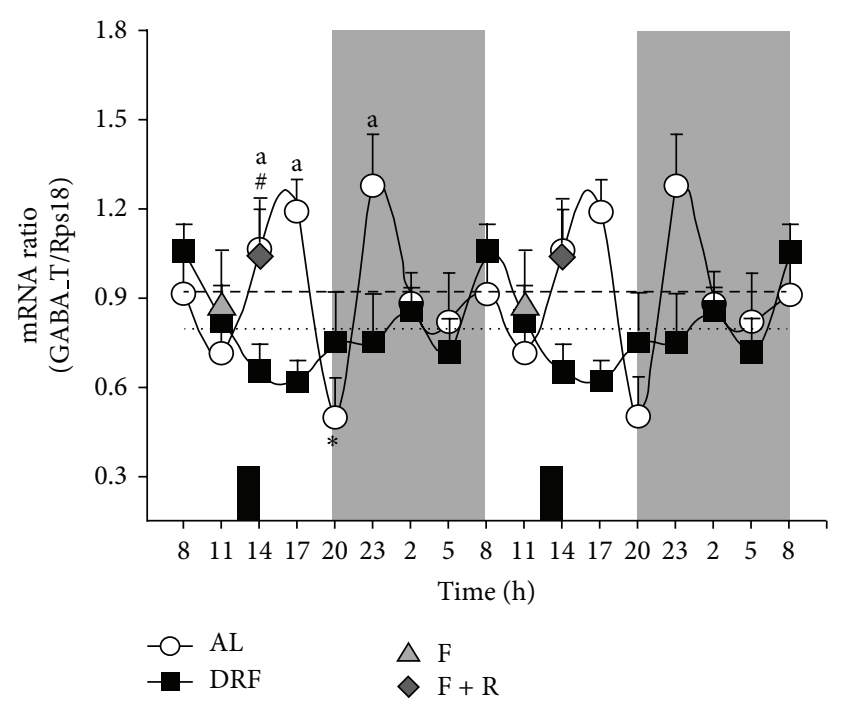

FIGURE 3: Analysis of the $24 \mathrm{~h}$ profile of relative mRNA expression of GABA-T in the liver of rats under AL and DRF conditions. Relative mRNA levels were determined by RT-qPCR and normalized to Rps18 expression. The shaded zone represents the dark phase. Food availability for DRF group is indicated by dark boxes (from 12:00 to $14: 00 \mathrm{~h}$ ). Graphs show the mean \pm SEM of 6 to 8 rats per time point. Average values are represented as a dashed line for the AL group and a dotted line for DRF group. Significant differences $(P<0.05)$ are indicated as follows: $*$ : DRF versus AL by oneway ANOVA and post hoc Bonferroni test, in both the homogenate and mitochondrial fraction; a: RFS versus AL by two-way ANOVA and post hoc Bonferroni test; \#: DRF versus $\mathrm{F}+\mathrm{R}$ at 14:00, by the Student's $t$-test.

the daily rhythmicity is modified by a new organization of the timing system $[29,30]$. The synchronization imposed by offering food for only $2-4 \mathrm{~h}$ per day is a powerful timing cue that, in some circumstances, overrules the control of the master pacemaker, the SCN [31]. This situation elicits food anticipatory activity as well as the expression of a biological clock (the FEO) that is independent of the SCN. The anatomical localization of the FEO is unknown, but the hypothesis guiding our research is that it is an emergent oscillator distributed among hypothalamic/midbrain nuclei that control food intake and peripheral organs such as liver, adrenals, stomach, and others important for nutrient processing $[15,32]$.

It is in the liver where the influence of the FEO is most evident. The liver plays central roles in both the processing of nutrients arriving from the duodenal tract and the modulating of the hunger-satiety cycle [33]. This fact is especially relevant during DRF because of the marked hyperphagia after the $2 \mathrm{~h}$ of food access. Indeed, the intense arrival of nutrients during the restricted feeding could be a factor in the daily entrainment showed by the liver [34].

In previous reports, we have shown that DRF/FEO expression strongly modifies the metabolic status of the liver mitochondria, promoting a more oxidized state and increasing the synthesis of ATP $[17,35]$. In addition, DRF/FEO expression is associated with an elevation of the malateaspartate shuttle activity [17]. Together these data indicate that liver mitochondria are responsive to the adaptations associated with FEO expression, as an example of the plasticity shown by the timing system during the metabolic adaptations associated with restricted feeding schedules. This is of particular interest to the present project since GABA-T is mostly a mitochondrial enzyme.

4.4. Rheostatic Adaptation. The exact molecular mechanisms that underlie the adaptive modifications of liver physiology during expression of the FEO and in response to the DRF protocol are not known. However, an initial rationale towards the identification of this sequence of events is to postulate that synchronizing circadian physiology by limited food access has an impact on the reciprocal regulation between energetic metabolic networks and the cellular timing system [36, 37]. During DRF/FEO expression, the connection between the molecular circadian clock and the metabolic activities has been thought to adopt a new relationship that is different from that in the control condition of ad libitum feeding. This new interaction has the characteristics of an emergent property, since it is not observed in the ad libitum condition or in the control groups of acute fasting and acute fasting followed by refeeding. For example, the increased amount GABA-T protein promoted by the protocol of DRF/FEO expression and detected by Western blot analysis (Figure 1) is unique and is observed neither in the AL group nor in the acute control. The same stands for the augmented GABA-T activity in liver homogenate and the loss of rhythmicity in GABA-T mRNA. Again, all these changes can be considered as part of the plastic adaptations that are present in the liver during the expression of the FEO.

One way to consider the emergence of new properties in biological systems is the concept of rheostasis [38], which is a term used in the theory of physiological control to describe regulation around shifting set points. In contrast to homeostasis, rheostasis triggers associations in situations with potentially adjustable settings [32]. In this context, expression of the FEO could involve a novel rheostatic adaptation in conditions in which two contradictory environmental temporal clues, light-dark cycle and DRF, are coexisting [39].

\section{Conclusions}

Biochemical properties of liver GABA-T were modified by the DRF protocol. An explanation for these findings should consider the coincidence of the FEO expression with a functional light-driven SCN. In addition, GABA-T regulation could be responding to the intense arrival of nutrients during the $2 \mathrm{~h}$ of food access, in the context of restricted feeding schedules. The data raise the possibility that alterations in the hepatic GABAergic system could be among the metabolic and physiological adaptions that occur in the liver during expression of the FEO. This possibility needs further exploration.

\section{Conflict of Interests}

The authors declare that there is no conflict of interests regarding the publication of this paper. 


\section{Authors' Contribution}

Dalia De Ita-Pérez, Isabel Méndez, and Olivia VázquezMartínez contributed equally to this project.

\section{Acknowledgments}

The authors wish to thank Dr. Dorothy Pless for reviewing the English of this paper and L.N. Fernando López Barrera for his help in preparing the figures. This study was supported by CONACyT (project 129-511) and DGAPA (projects IN202412, MDM, and IA200713, IM) from PAPIIT, UNAM, México.

\section{References}

[1] P. K. Mehta, T. I. Hale, and P. Christen, "Aminotransferases: demonstration of homology and division into evolutionary subgroups," European Journal of Biochemistry, vol. 214, no. 2, pp. 549-561, 1993.

[2] N. Bouché, B. Lacombe, and H. Fromm, "GABA signaling: a conserved and ubiquitous mechanism," Trends in Cell Biology, vol. 13, no. 12, pp. 607-610, 2003.

[3] D. J. Garry, H. D. Coulter, and T. J. McIntee, "Immunoreactive GABA transaminase within the pancreatic islet is localized in mitochondria of the B-cell," Journal of Histochemistry and Cytochemistry, vol. 35, no. 8, pp. 831-836, 1987.

[4] P. Storici, G. Capitani, D. de Biase et al., "Crystal structure of GABA-aminotransferase, a target for antiepileptic drug therapy," Biochemistry, vol. 38, no. 27, pp. 8628-8634, 1999.

[5] P. Storici, D. De Biase, F. Bossa et al., "Structures of $\gamma$ aminobutyric acid (GABA) aminotransferase, a pyridoxal 5/phosphate, and [2Fe-2S] cluster-containing enzyme, complexed with $\gamma$-ethynyl-GABA and with the antiepilepsy drug vigabatrin," Journal of Biological Chemistry, vol. 279, no. 1, pp. 363-373, 2004.

[6] Y. Kontani, S. F. Sakata, K. Matsuda, T. Ohyama, K. Sano, and N. Tamaki, "The mature size of rat 4-aminobutyrate aminotransferase is different in liver and brain," European Journal of Biochemistry, vol. 264, no. 1, pp. 218-222, 1999.

[7] I. K. Franklin and C. B. Wollheim, "GABA in the endocrine pancreas: its putative role as an islet cell paracrine-signalling molecule," Journal of General Physiology, vol. 123, no. 3, pp. 185190, 2004.

[8] A. Gladkevich, J. Korf, V. P. Hakobyan, and K. V. Melkonyan, "The peripheral GABAergic system as a target in endocrine disorders," Autonomic Neuroscience: Basic and Clinical, vol. 124, no. 1-2, pp. 1-8, 2006.

[9] R. Erlitzki, Y. Gong, M. Zhang, and G. Minuk, "Identification of $\gamma$-aminobutyric acid receptor subunit types in human and rat liver," The American Journal of Physiology: Gastrointestinal and Liver Physiology, vol. 279, no. 4, pp. G733-G739, 2000.

[10] G. Y. Minuk, "Gamma-aminobutyric acid and the liver," Digestive Diseases, vol. 11, no. 1, pp. 45-54, 1993.

[11] Y. H. Li, Y. D. Liu, Y. D. Li et al., "GABA stimulates human hepatocellular carcinoma growth through overexpressed GABAA receptor theta subunit," World Journal of Gastroenterology, vol. 18, no. 21, pp. 2704-2711, 2012.

[12] J. Y. Wu, L. G. Moss, and O. Chude, "Distribution and tissue specificity of 4-aminobutyrate-2-oxoglutarate aminotransferase," Neurochemical Research, vol. 3, no. 2, pp. 207-219, 1978.
[13] R. Dhakal, V. K. Bajpai, and K. H. Baek, "Production of gaba (gamma-Aminobutyric acid) by microorganisms: a review," Brazilian Journal of Microbiology, vol. 43, no. 4, pp. 1230-1241, 2012.

[14] F. K. Stephan, “The "other” circadian system: food as a zeitgeber," Journal of Biological Rhythms, vol. 17, no. 4, pp. 284-292, 2002.

[15] B. T. S. Carneiro and J. F. Araujo, "The food-entrainable oscillator: a network of interconnected brain structures entrained by humoral signals?" Chronobiology International, vol. 26, no. 7, pp. 1273-1289, 2009.

[16] R. E. Mistlberger, "Neurobiology of food anticipatory circadian rhythms," Physiology and Behavior, vol. 104, no. 4, pp. 535-545, 2011.

[17] A. Báez-Ruiz, C. Escobar, R. Aguilar-Roblero, O. VázquezMartínez, and M. Díaz-Muñoz, "Metabolic adaptations of liver mitochondria during restricted feeding schedules," The American Journal of Physiology: Gastrointestinal and Liver Physiology, vol. 289, no. 6, pp. G1015-G1023, 2005.

[18] C. Molina-Aguilar, J. Pérez-Sánchez, O. Vázquez-Martínez, J. Rivera-Zavala, and M. Díaz-Muñoz, "Restricted food access during the daytime modifies the 24-h rhythmicity of apoptosis and cellular duplication in rat liver," Biological Rhythm Research, vol. 43, no. 1, pp. 25-37, 2012.

[19] F. Portaluppi, Y. Touitou, and M. H. Smolensky, "Ethical and methodological standards for laboratory and medical biological rhythm research," Chronobiology International, vol. 25, no. 6, pp. 999-1016, 2008.

[20] M. Ángeles-Castellanos, J. Mendoza, M. Díaz-Muñoz, and C. Escobar, "Food entrainment modifies the c-Fos expression pattern in brain stem nuclei of rats," The American Journal of Physiology: Regulatory Integrative and Comparative Physiology, vol. 288, no. 3, pp. R678-R684, 2005.

[21] I. Aguilar-Delfín, F. López-Barrera, and R. Hernández-Muñoz, "Selective enhancement of lipid peroxidation in plasma membrane in two experimental models of liver regeneration: partial hepatectomy and acute CC14administration," Hepatology, vol. 24, no. 3, pp. 657-662, 1996.

[22] M. M. Bradford, "A rapid and sensitive method for the quantitation of microgram quantities of protein utilizing the principle of protein dye binding," Analytical Biochemistry, vol. 72, no. 1-2, pp. 248-254, 1976.

[23] M. J. Jung, B. Lippert, and B. W. Metcalf, "The effect of 4 amino hex 5 ynoic acid ( $\gamma$ acetylenic GABA, $\gamma$ ethynyl GABA) a catalytic inhibitor of GABA transaminase, on brain GABA metabolism in vivo," Journal of Neurochemistry, vol. 28, no. 4, pp. 717-723, 1977.

[24] P. Zuther, S. Gorbey, and B. Lemmer, "Chronos-Fit v 1. 06," 2009, http://www.ma.uni-heidelberg.de/inst/phar/lehre/ chrono.html.

[25] B. E. Faulkner-Jones, D. S. Cram, J. Kun, and L. C. Harrison, "Localization and quantitation of expression of two glutamate decarboxylase genes in pancreatic $\beta$-cells and other peripheral tissues of mouse and rat," Endocrinology, vol. 133, no. 6, pp. 2962-2972, 1993.

[26] C. Lodewyks, J. Rodriguez, J. Yan et al., "GABA-B receptor activation inhibits the in vitro migration of malignant hepatocytes," Canadian Journal of Physiology and Pharmacology, vol. 89, no. 6, pp. 393-400, 2011.

[27] Y. Zhou, S. Holmseth, C. Guo et al., "Deletion of the gammaaminobutyric acid transporter 2 (GAT2 and SLC6A13) gene 
in mice leads to changes in liver and brain taurine contents," Journal of Biological Chemistry, vol. 287, no. 42, pp. 3573335746, 2012.

[28] D. M. Treiman, "GABAergic mechanisms in epilepsy," Epilepsia, vol. 42, 3, pp. 8-12, 2001.

[29] C. Dibner, U. Schibler, and U. Albrecht, "The mammalian circadian timing system: organization and coordination of central and peripheral clocks," Annual Review of Physiology, vol. 72, pp. 517-549, 2009.

[30] I. Schmutz, U. Albrecht, and J. A. Ripperger, "The role of clock genes and rhythmicity in the liver," Molecular and Cellular Endocrinology, vol. 349, no. 1, pp. 38-44, 2012.

[31] C. A. Feillet, U. Albrecht, and E. Challet, "Feeding time for the brain: a matter of clocks," Journal of Physiology Paris, vol. 100, no. 5-6, pp. 252-260, 2006.

[32] R. Aguilar-roblero and M. Díaz-muñoz, "Chronostatic adaptations in the liver to restricted feeding: the FEO as an emergent oscillator," Sleep and Biological Rhythms, vol. 8, no. 1, pp. 9-17, 2010.

[33] P. Magni, E. Dozio, M. Ruscica et al., "Feeding behavior in mammals including humans," Annals of the New York Academy of Sciences, vol. 1163, pp. 221-232, 2009.

[34] J. Mendoza, "Circadian clocks: setting time by food," Journal of Neuroendocrinology, vol. 19, no. 2, pp. 127-137, 2007.

[35] M. Díaz-Muñoz, O. Vázquez-Martínez, R. Aguilar-Roblero, and C. Escobar, "Anticipatory changes in liver metabolism and entrainment of insulin, glucagon, and corticosterone in foodrestricted rats," The American Journal of Physiology: Regulatory Integrative and Comparative Physiology, vol. 279, no. 6, pp. R2048-R2056, 2000.

[36] R. Buijs, R. Salgado, E. Sabath, and C. Escobar, "Peripheral circadian oscillators: time and food," Progress in MolecularBiology and Translational Sciences, vol. 119, pp. 83-103, 2013.

[37] U. Albrecht, "Timing to perfection: the biology of central and peripheral circadian clocks," Neuron, vol. 74, no. 2, pp. 246-260, 2012.

[38] N. Mrosovsky, Rheostasis: the Physiology of Change, Oxord University Press, 1990.

[39] B. Kornmann, O. Schaad, H. Bujard, J. S. Takahashi, and U. Schibler, "System-driven and oscillator-dependent circadian transcription in mice with a conditionally active liver clock," PLoS Biology, vol. 5, no. 2, article e34, 2007. 

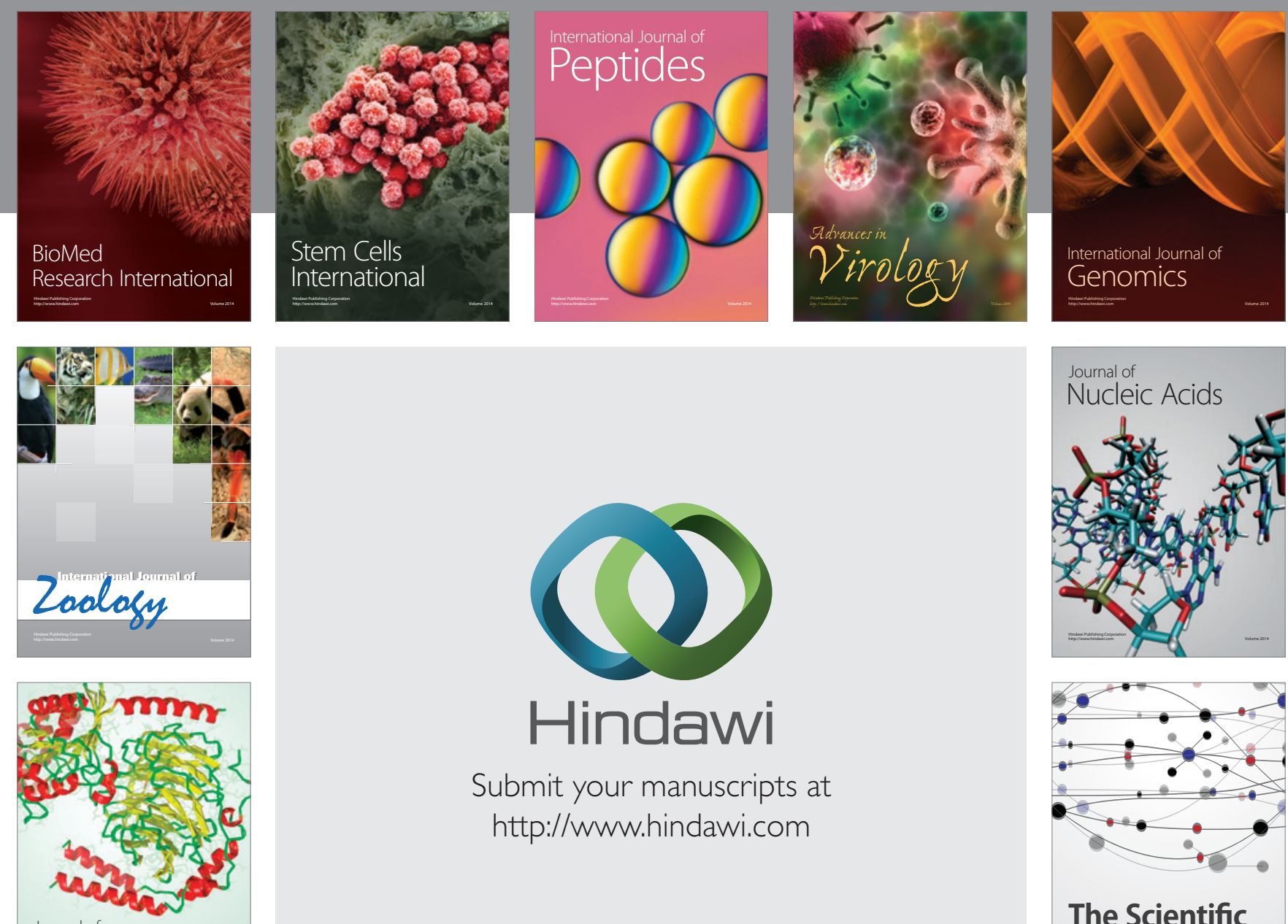

Submit your manuscripts at

http://www.hindawi.com

Journal of
Signal Transduction
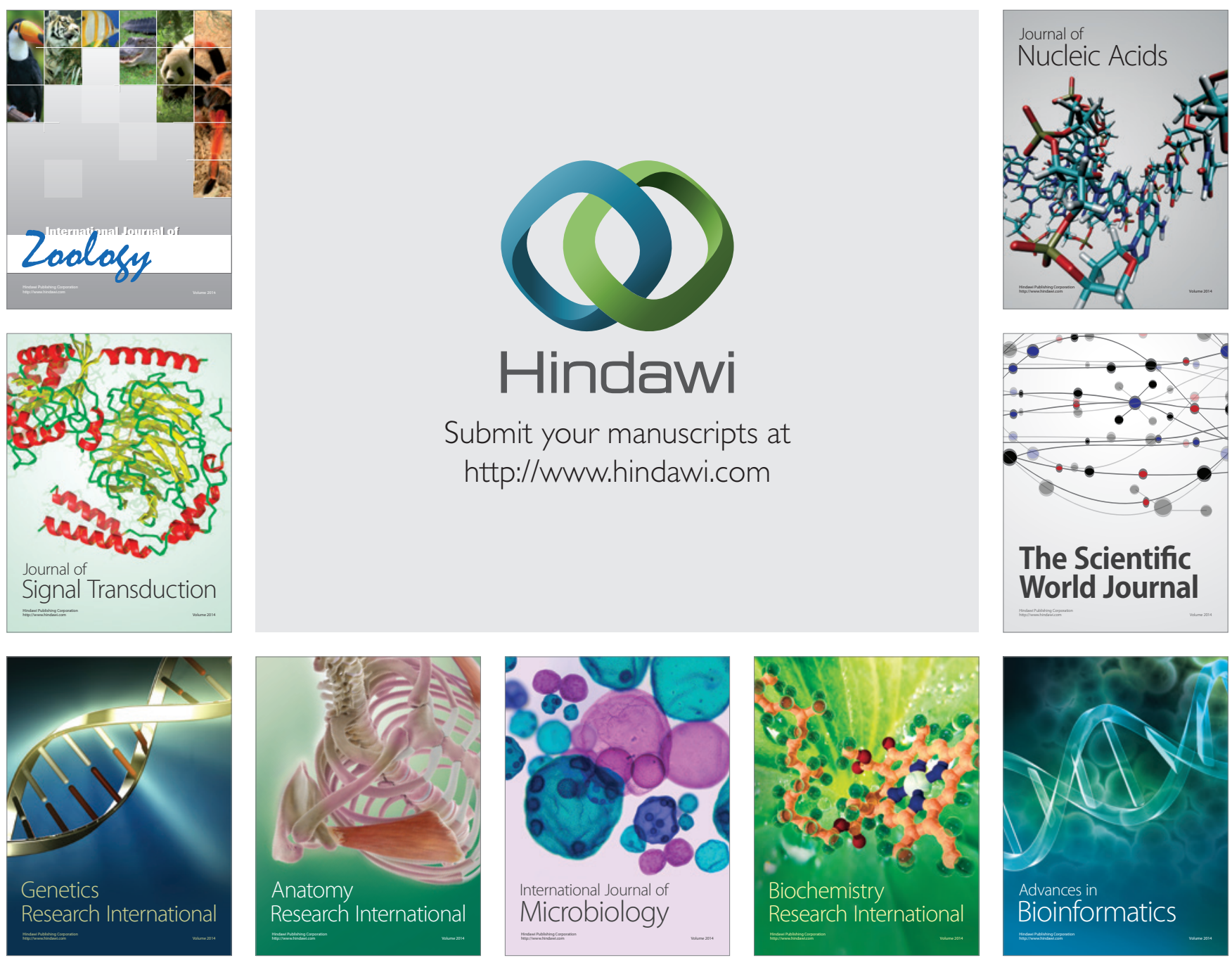

The Scientific World Journal
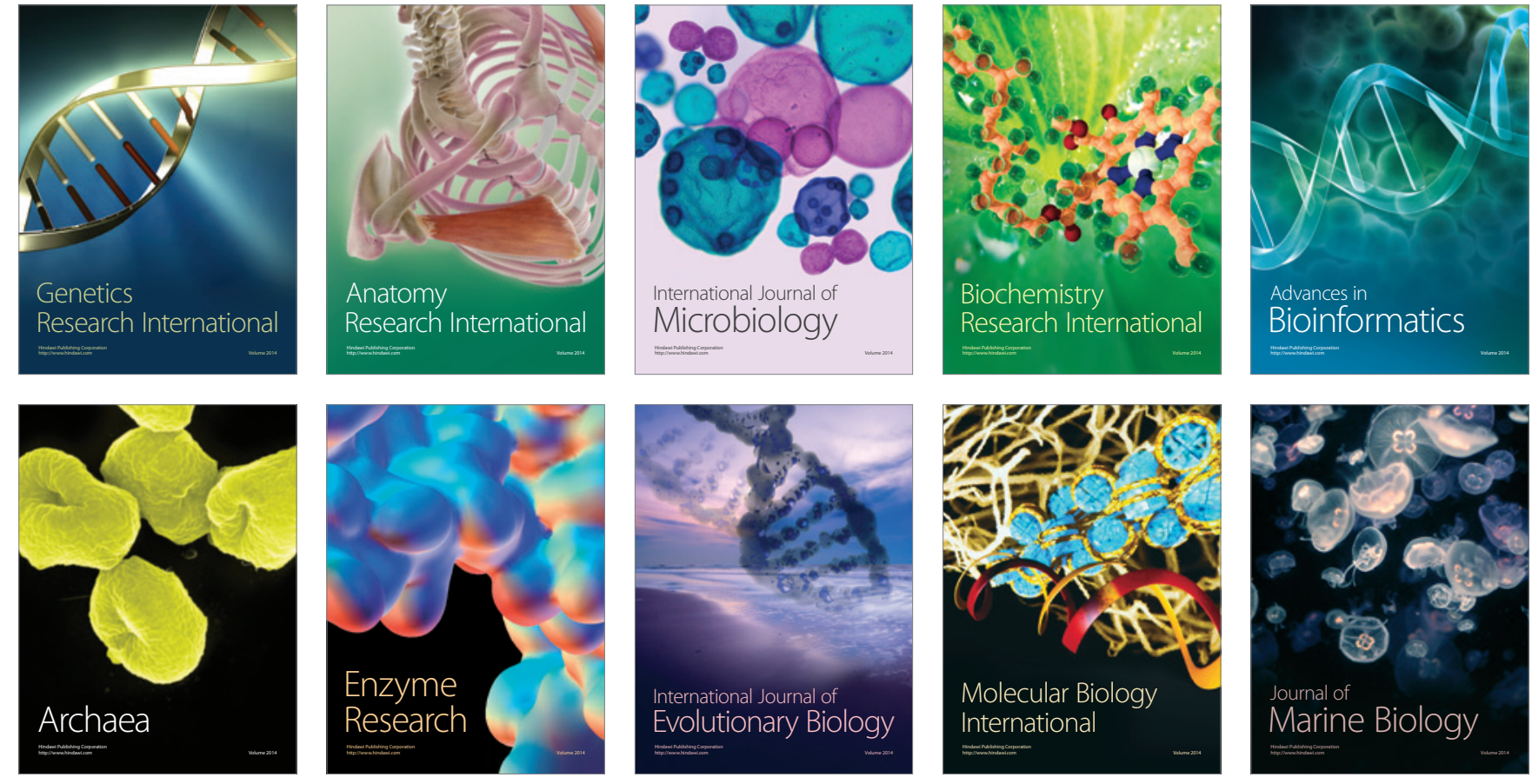